\title{
OPTIMALITY CONDITIONS FOR IRREGULAR INEQUALITY-CONSTRAINED PROBLEMS*
}

\author{
A. F. IZMAILOV ${ }^{\dagger}$ AND M. V. SOLODOV
}

\begin{abstract}
We consider feasible sets given by conic constraints, where the cone defining the constraints is convex with nonempty interior. We study the case where the feasible set is not assumed to be regular in the classical sense of Robinson and obtain a constructive description of the tangent cone under a certain new second-order regularity condition. This condition contains classical regularity as a special case, while being weaker when constraints are twice differentiable. Assuming that the cone defining the constraints is finitely generated, we also derive a special form of primal-dual optimality conditions for the corresponding constrained optimization problem. Our results subsume optimality conditions for both the classical regular and second-order regular cases, while still being meaningful in the more general setting in the sense that the multiplier associated with the objective function is nonzero.
\end{abstract}

Key words. tangent cone, regularity, constraint qualification, optimality conditions

AMS subject classifications. 90C30, 46T20, 47J07, 90C33

PII. S0363012999357549

1. Introduction. Let $X$ and $Y$ be normed linear spaces. We consider the sets given by

$$
D=\{x \in X \mid F(x) \in K\},
$$

where the constraint mapping $F: X \rightarrow Y$ is smooth enough and $K$ is a closed convex cone in $Y$ with nonempty interior. The problem of an accurate and constructive description of the tangent cone to a set at a given point is fundamental for many reasons, one of which is deriving optimality conditions. Recall that a vector $h \in X$ is called tangent to a set $D \subset X$ at a point $\bar{x} \in D$ if there exists a mapping $r: \Re_{+} \rightarrow X$ such that

$$
\bar{x}+t h+r(t) \in D \quad \forall t \in \Re_{+}, \quad\|r(t)\|=o(t) .
$$

The set of all such vectors $h$ in $X$ is the tangent cone to the set $D$ at the point $\bar{x}$, which we shall denote by $T_{D}(\bar{x})$. As is well known,

$$
T_{D}(\bar{x}) \subset\left\{h \in X \mid F^{\prime}(\bar{x}) h \in T_{K}(F(\bar{x}))\right\},
$$

which is the first-order necessary condition for tangency. To obtain a precise description of $T_{D}(\bar{x})$, i.e., a sufficient condition for tangency, some regularity (also called constraint qualification) condition is needed. One classical condition in this setting is Robinson's condition [27]:

$$
0 \in \operatorname{int}\left(F(\bar{x})+\operatorname{Im} F^{\prime}(\bar{x})-K\right) .
$$

*Received by the editors June 7, 1999; accepted for publication (in revised form) June 8, 2001; published electronically December 7, 2001.

http://www.siam.org/journals/sicon/40-4/35754.html

${ }^{\dagger}$ Computing Center of the Russian Academy of Sciences, Vavilova Str. 40, Moscow, GSP-1, Russia (izmaf@ccas.ru). The research of this author was supported by Russian Foundation for Basic Research grants 99-01-00472 and 01-01-00810.

${ }^{\ddagger}$ Instituto de Matemática Pura e Aplicada, Estrada Dona Castorina 110, Jardim Botânico, Rio de Janeiro, RJ 22460-320, Brazil (solodov@impa.br). The research of this author was supported in part by CNPq grant 300734/95-6, by PRONEX-Optimization, and by FAPERJ. 
Note that in (1.4) cone $K$ is not required to have a nonempty interior. If (1.4) is satisfied, then (1.3) holds as an equality, e.g., [12, Corollary 2.91]. Deriving an accurate constructive description of the tangent cone without assuming (1.4) and, more generally, when (1.3) does not necessarily hold as an equality, is one of the principal goals of this paper. Our approach is based on a certain new notion of second-order regularity, which in the setting of $K$ with nonempty interior is weaker than (1.4); see Definition 2.1 and Remark 2.1. An immediate application of this description is the primal form of necessary optimality conditions for the problem

$$
\min \{f(x) \mid x \in D\},
$$

where the objective function $f: X \rightarrow \Re$ is smooth enough.

Our second goal is to obtain primal-dual optimality conditions for the irregular case, with a nonzero multiplier associated to the objective function. If $\bar{x}$ is a local solution of (1.5), (1.1), then the classical F. John-type first-order necessary optimality conditions (e.g., see [11]) state that there exists a generalized Lagrange multiplier $\left(y_{0}, y^{*}\right) \in\left(\Re \times Y^{*}\right) \backslash\{0\}$ such that

$$
\begin{aligned}
& y_{0} f^{\prime}(\bar{x})-\left(F^{\prime}(\bar{x})\right)^{*} y^{*}=0, \\
& F(\bar{x}) \in K, y^{*} \in K^{*},\left\langle y^{*}, F(\bar{x})\right\rangle=0, y_{0} \geq 0,
\end{aligned}
$$

where $Y^{*}$ is the dual space of $Y,\left(F^{\prime}(\bar{x})\right)^{*}$ is the adjoint operator of $F^{\prime}(\bar{x})$, and $K^{*}$ is the dual cone of $K$. If $y_{0}=0$, the F. John conditions hold trivially independently of the objective function and therefore their utility for describing optimality in that case is very limited (at least without some further developments). Assumptions that guarantee the existence of a multiplier $\left(y_{0}, y^{*}\right)$ with $y_{0} \neq 0$ are again constraint qualification conditions, such as (1.4). For problems with a finitely generated cone $K$, without assuming (1.4) or equality in (1.3), we obtain a special form of primal-dual optimality conditions under our assumption of second-order regularity. Our optimality conditions resemble the structure of (1.6), where $y_{0} \neq 0$ and a certain term involving the second derivative of $F$ is added to the standard Lagrangian; see Theorem 3.2. Our optimality conditions subsume those for the classical regular case of (1.4), as well as those for the more general second-order regular case of $[7,8]$; see section 4 .

In section 4 , we compare our results with other approaches relevant for irregular inequality-constrained problems. We also provide an example showing that our results can be used to verify optimality in cases where other known approaches appear not to be applicable. We note that those cases do not seem pathological or exotic; see Example 4.1.

Finally, we note that in the case of the nonlinear programming problem, i.e., when $Y=\Re^{m} \times \Re^{s}$ and $K=\Re_{-}^{m} \times\{0\}$, Robinson's regularity condition (1.4) reduces to the classical Mangasarian-Fromovitz constraint qualification [23], and with $y_{0} \neq 0$ optimality conditions (1.6) become the classical Karush-Kuhn-Tucker conditions.

Our notation is fairly standard. If $\Sigma$ is a topological linear space, then $\Sigma^{*}$ denotes its (topologically) dual space and $\langle\cdot, \cdot\rangle$ is the pairing of elements in $\Sigma^{*}$ and $\Sigma$, i.e., $\left\langle\sigma^{*}, \sigma\right\rangle$ is the value of the linear functional $\sigma^{*} \in \Sigma^{*}$ on $\sigma \in \Sigma$. For a cone $C$ in $\Sigma$, the positive dual cone (sometimes also referred to as the polar cone) of $C$ is $C^{*}:=\left\{\sigma^{*} \in \Sigma^{*} \mid\left\langle\sigma^{*}, \sigma\right\rangle \geq 0 \quad \forall \sigma \in C\right\}$. For an arbitrary set $\Omega$ in $\Sigma$, the set orthogonal to $\Omega$ is $\Omega^{\perp}:=\left\{\sigma^{*} \in \Sigma^{*} \mid\left\langle\sigma^{*}, \sigma\right\rangle=0 \quad \forall \sigma \in \Omega\right\}$. If $\Upsilon$ and $\Sigma$ are topological linear spaces and $\Lambda: \Upsilon \rightarrow \Sigma$ is a continuous linear operator, then $\Lambda^{*}$ : $\Sigma^{*} \rightarrow \Upsilon^{*}$ denotes the adjoint operator of $\Lambda$. The interior and the closure of a set $\Omega$ (in appropriate topology) are denoted by int $\Omega$ and $\operatorname{cl} \Omega$, respectively, and linear and 
conic hulls of this set (in appropriate linear space) by $\operatorname{lin} \Omega$ and cone $\Omega$, respectively. A cone $C$ in a linear space $\Sigma$ is referred to as finitely generated if either it is empty or there exists a positive integer $s$ and some elements $\sigma^{i} \in \Sigma, i=1, \ldots, s$, such that $\operatorname{cl} C=\operatorname{cone}\left\{\sigma^{1}, \ldots, \sigma^{s}\right\} \cup\{0\}$. When we write that a mapping $F$ is twice Fréchetdifferentiable at a point $\bar{x}$, we mean that it is Fréchet-differentiable on a neighborhood of $\bar{x}$, and its derivative is Fréchet-differentiable at $\bar{x}$ (and similarly for higher-order Fréchet-differentiability).

Some auxiliary facts from convex analysis that are used throughout the paper are collected in the appendix.

2. Tangent cone description. As is well known [24], [12, Lemma 2.99], in our setting where int $K \neq \emptyset$, Robinson's regularity condition (1.4) is equivalent to

$$
\exists \bar{\xi} \in X \text { such that } F(\bar{x})+F^{\prime}(\bar{x}) \bar{\xi} \in \operatorname{int} K .
$$

This condition implies that for $h \in T_{D}(\bar{x})$ the inclusion

$$
F^{\prime}(\bar{x}) h \in T_{K}(F(\bar{x}))=\operatorname{cl}(K+\operatorname{lin}\{F(\bar{x})\})
$$

is both necessary and sufficient, e.g., [12, Corollary 2.91]. In the irregular case, $T_{D}(\bar{x})$ can be smaller than the set of $h \in X$ satisfying (2.2), and a more refined description is needed. To this end, it is natural to take into account the second-order information about $F$ at $\bar{x}$. We proceed with a second-order characterization of the tangent cone, starting with the following definition.

DEFINITION 2.1. We say that conic constraints in (1.1) are second-order regular at a feasible point $\bar{x}$ with respect to a direction $h \in X$ if

$$
\begin{aligned}
& \exists(\bar{\xi}, \bar{h}) \in X \times X \text { such that } F(\bar{x})+F^{\prime}(\bar{x}) \bar{h} \in K, \\
& \qquad F(\bar{x})+F^{\prime}(\bar{x}) \bar{\xi}+F^{\prime \prime}(\bar{x})[h, \bar{h}] \in \operatorname{int} K .
\end{aligned}
$$

Remark 2.1. If Robinson's condition (2.1) is satisfied, then second-order regularity holds with respect to every $h \in X$, including $h=0$. (To verify this, just choose $\bar{\xi}$ satisfying $(2.1)$ and $\bar{h}=0$.)

Observe further that Definition 2.1 is equivalent to saying that

$$
\begin{aligned}
\exists \bar{h} \in X \text { such that } & F^{\prime}(\bar{x}) \bar{h} \in T_{K}^{r}(F(\bar{x}))=K+\operatorname{lin}\{F(\bar{x})\}, \\
& F^{\prime \prime}(\bar{x})[h, \bar{h}] \in \operatorname{int} K+\operatorname{lin}\{F(\bar{x})\}+\operatorname{Im} F^{\prime}(\bar{x}),
\end{aligned}
$$

where $T_{K}^{r}(y)$ stands for the so-called radial tangent cone to $K$ at $y \in K$. This form of second-order regularity will be used in the subsequent analysis. We are now ready to state the main result of this section.

TheOrem 2.2. Let $X$ and $Y$ be normed linear spaces and let $K$ be a closed convex cone in $Y$ with a nonempty interior. Let set $D$ be given by (1.1), where $F: X \rightarrow Y$ is twice Fréchet-differentiable at a point $\bar{x} \in D$. Then the following statements hold.

1. Every $h \in T_{D}(\bar{x})$ satisfies (2.2) as well as the following condition:

$$
F^{\prime \prime}(\bar{x})[h]^{2} \in \operatorname{cl}\left(K+\operatorname{lin}\{F(\bar{x})\}+\operatorname{Im} F^{\prime}(\bar{x})\right) .
$$

2. If $h \in X$ satisfies

$$
F^{\prime}(\bar{x}) h \in K+\operatorname{lin}\{F(\bar{x})\}
$$

and (2.5), and if constraints in (1.1) are second-order regular at $\bar{x}$ with respect to this $h$, then $h \in T_{D}(\bar{x})$. 
Proof. Take an arbitrary $h \in T_{D}(\bar{x})$. Relation (2.2) is standard, so we have to prove only (2.5). By twice differentiability of $F$, for every $t>0$ we have that

$$
\begin{aligned}
\frac{1}{2} F^{\prime \prime}(\bar{x})[t h]^{2}= & F(\bar{x}+t h+r(t))-F(\bar{x})-F^{\prime}(\bar{x})(t h+r(t)) \\
& -\frac{1}{2} F^{\prime \prime}(\bar{x})[r(t)]^{2}-F^{\prime \prime}(\bar{x})[t h, r(t)]+\omega_{2}(t),
\end{aligned}
$$

where $\omega_{2}: \Re_{+} \rightarrow Y,\left\|\omega_{2}(t)\right\|=o\left(t^{2}\right)$. Observe that the first term in the right-hand side is in $K$ due to (1.2), the second is in $\operatorname{lin}\{F(\bar{x})\}$, and the third is $\operatorname{in} F^{\prime}(\bar{x})$. Dividing by $t^{2}$ and passing onto the limit as $t \rightarrow 0+$, we obtain (2.5).

Assume now that some $h \in X$ satisfies (2.6) and (2.5). Then there exist $y_{1} \in K$ and $\lambda_{1} \in \Re$ such that $F^{\prime}(\bar{x}) h=y_{1}+\lambda_{1} F(\bar{x})$. Consider first the case where

$$
F^{\prime \prime}(\bar{x})[h]^{2} \in \operatorname{int} K+\operatorname{lin}\{F(\bar{x})\}+\operatorname{Im} F^{\prime}(\bar{x}),
$$

so that there exist $y_{2} \in \operatorname{int} K, \lambda_{2} \in \Re$, and $x \in X$ such that $F^{\prime \prime}(\bar{x})[h]^{2}=y_{2}+\lambda_{2} F(\bar{x})+$ $F^{\prime}(\bar{x}) x$. In that case, we obtain that

$$
\begin{aligned}
F\left(\bar{x}+t h-\frac{t^{2}}{2} x\right)= & F(\bar{x})+F^{\prime}(\bar{x})\left(t h-\frac{t^{2}}{2} x\right) \\
& +\frac{1}{2} F^{\prime \prime}(\bar{x})\left[t h-\frac{t^{2}}{2} x\right]^{2}+\omega_{2}(t) \\
= & F(\bar{x})+t\left(y_{1}+\lambda_{1} F(\bar{x})\right)-\frac{t^{2}}{2} F^{\prime}(\bar{x}) x \\
& +\frac{t^{2}}{2}\left(y_{2}+\lambda_{2} F(\bar{x})+F^{\prime}(\bar{x}) x\right)+\omega_{2}(t) \\
= & \left(1+t \lambda_{1}+\frac{t^{2}}{2} \lambda_{2}\right) F(\bar{x})+t y_{1}+\frac{t^{2}}{2} y_{2}+\omega_{2}(t) \\
\in & \operatorname{int} K,
\end{aligned}
$$

where $\omega_{2}: \Re_{+} \rightarrow Y,\left\|\omega_{2}(t)\right\|=o\left(t^{2}\right)$, and the inclusion follows from Lemma A.5 for every $t>0$ sufficiently small. In particular, we conclude that if $(2.7)$ holds, then $h \in T_{D}(\bar{x})$.

If (2.7) does not hold, but there exists a sequence $\left\{h^{k}\right\} \subset X$ converging to $h$ such that (2.7) is satisfied for every element of this sequence, then again $h \in T_{D}(\bar{x})$ by the closedness of $T_{D}(\bar{x})$. We proceed to explicitly construct the desired sequence $\left\{h^{k}\right\}$ under the hypothesis of the theorem that there exists an element $\bar{h} \in X$ for which (2.3), (2.4) are satisfied. Let us take $h^{k}=(1-1 / k) h+\bar{h} / k, k=1,2, \ldots$. For each index $k$ we then obtain

$$
F^{\prime}(\bar{x}) h^{k}=\left(1-\frac{1}{k}\right) F^{\prime}(\bar{x}) h+\frac{1}{k} F^{\prime}(\bar{x}) \bar{h} \in K+\operatorname{lin}\{F(\bar{x})\},
$$

where the inclusion follows from (2.6), (2.3). We further obtain

$$
\begin{aligned}
F^{\prime \prime}(\bar{x})\left[h^{k}\right]^{2}= & \left(1-\frac{1}{k}\right)^{2} F^{\prime \prime}(\bar{x})[h]^{2} \\
& +\frac{1}{k}\left(2\left(1-\frac{1}{k}\right) F^{\prime \prime}(\bar{x})[h, \bar{h}]+\frac{1}{k} F^{\prime \prime}(\bar{x})[\bar{h}]^{2}\right) \\
\in & \operatorname{int} K+\operatorname{lin}\{F(\bar{x})\}+\operatorname{Im} F^{\prime}(\bar{x}),
\end{aligned}
$$


where the inclusion holds for all $k$ sufficiently large, due to (2.4), (2.5) and Lemmas A.2 and A.5. This construction completes the proof.

In section 4, we compare this theorem (as well as the other results of this paper) with related facts and approaches to irregular inequality constraints and provide an illustrative example. Here, we note that in the regular case (1.4) implies that

$$
K+\operatorname{lin}\{F(\bar{x})\}+\operatorname{Im} F^{\prime}(\bar{x})=Y,
$$

and thus (2.5) holds trivially for every $h \in X$. This observation together with Remark 2.1 show that Theorem 2.2 subsumes (when $K$ has nonempty interior) the classical result on the tangent cone in the regular case. In the irregular case, the right-hand side of (2.5) does not coincide with $Y$ (again, in our setting of int $K \neq \emptyset$ ), and therefore condition (2.5) is nontrivial.

Remark 2.2. If $K$ is a finitely generated cone, then (2.6) is equivalent to (2.2), as the right-hand sides of these relations coincide (this follows from Lemma A.3). But in the general case, one cannot substitute the weaker condition (2.2) into the sufficiency part of the theorem, as illustrated by the following example.

Example 2.1. Let $X=\Re, Y=\Re^{3}$, and

$$
\begin{gathered}
K=\operatorname{cone}\left\{\left.y \in \Re^{3}\left|y_{1}=1, y_{3}=\right| y_{2}\right|^{3 / 2}\right\}, \\
F: \Re \rightarrow \Re^{3}, \quad F(x)=\left(1, x, x^{2}\right) .
\end{gathered}
$$

For the point $\bar{x}=0 \in \Re$, we have $F(0) \in K, \operatorname{cl}(K+\operatorname{lin}\{F(\bar{x})\})=\operatorname{cl}(K+\operatorname{lin}\{F(\bar{x})\}+$ $\left.\operatorname{Im} F^{\prime}(\bar{x})\right)=\left\{y \in \Re^{3} \mid y_{3} \geq 0\right\}$, and, as is easy to see, for element $h=1$ conditions (2.3), (2.4) hold with $\bar{h}=h$. At the same time, 0 is obviously an isolated point of the set $D$ given by (1.1), and hence $T_{D}(\bar{x})=\{0\}$.

3. Optimality conditions. We now turn our attention to the optimization problem (1.5), where the feasible set is given by (1.1). We assume that $K$ is a closed convex cone with nonempty interior (for primal-dual optimality conditions, also finitely generated), the objective function $f$ is Fréchet-differentiable at the point $\bar{x} \in D$ under consideration, and the mapping $F$ is twice Fréchet-differentiable at $\bar{x}$.

Following the developments of section 2, we first introduce some relevant cones. Let $H_{2}(\bar{x})$ be the set of all elements satisfying the second-order necessary conditions of tangency $(2.2),(2.5)$ stated in Theorem 2.2, i.e.,

$$
H_{2}(\bar{x}):=\left\{\begin{array}{l|l}
h \in X & \begin{array}{l}
F^{\prime}(\bar{x}) h \in T_{K}(F(\bar{x}))=\operatorname{cl}(K+\operatorname{lin}\{F(\bar{x})\}) \\
F^{\prime \prime}(\bar{x})[h]^{2} \in \operatorname{cl}\left(K+\operatorname{lin}\{F(\bar{x})\}+\operatorname{Im} F^{\prime}(\bar{x})\right)
\end{array}
\end{array}\right\},
$$

and $\tilde{H}_{2}(\bar{x})$ be the set of elements satisfying the two relations (2.6) and (2.5), which appear in the sufficiency part:

$$
\tilde{H}_{2}(\bar{x}):=\left\{\begin{array}{l|l}
h \in X & \begin{array}{l}
F^{\prime}(\bar{x}) h \in T_{K}^{r}(F(\bar{x}))=K+\operatorname{lin}\{F(\bar{x})\} \\
F^{\prime \prime}(\bar{x})[h]^{2} \in \operatorname{cl}\left(K+\operatorname{lin}\{F(\bar{x})\}+\operatorname{Im} F^{\prime}(\bar{x})\right)
\end{array}
\end{array}\right\} .
$$

Finally, let $\bar{H}_{2}(\bar{x})$ consist of all elements satisfying the sufficient conditions of tangency stated in Theorem 2.2, i.e.,

$$
\bar{H}_{2}(\bar{x}):=\left\{h \in \tilde{H}_{2}(\bar{x}) \mid \exists \bar{h} \in X: \begin{array}{l}
F^{\prime}(\bar{x}) \bar{h} \in K+\operatorname{lin}\{F(\bar{x})\} \\
F^{\prime \prime}(\bar{x})[h, \bar{h}] \in \operatorname{int} K+\operatorname{lin}\{F(\bar{x})\}+\operatorname{Im} F^{\prime}(\bar{x})
\end{array}\right\} .
$$

By these definitions,

$$
\bar{H}_{2}(\bar{x}) \cup\{0\} \subset \tilde{H}_{2}(\bar{x}) \subset H_{2}(\bar{x}) .
$$


Note that if the second-order regularity condition holds with respect to all $h \in \tilde{H}_{2}(\bar{x}) \backslash$ $\{0\}$, then the first inclusion in (3.1) holds as an equality. If cone $K$ is finitely generated, then the second inclusion is also an equality (recall Remark 2.2). By Theorem 2.2, we also have that

$$
\bar{H}_{2}(\bar{x}) \cup\{0\} \subset T_{D}(\bar{x}) \subset H_{2}(\bar{x}) .
$$

If $K$ is finitely generated and the second-order regularity condition holds with respect to all $h \in \tilde{H}_{2}(\bar{x}) \backslash\{0\}$, then we have equalities throughout (3.2).

The left-hand inclusion in (3.2) immediately implies the following primal necessary optimality condition for our problem.

Theorem 3.1. Let $X$ and $Y$ be normed linear spaces, and let $K$ be a closed convex cone in $Y$ with a nonempty interior. Assume that $f: X \rightarrow \Re$ is Fréchetdifferentiable, and $F: X \rightarrow Y$ is twice Fréchet-differentiable at a point $\bar{x} \in D$, where $D$ is given by (1.1). If $\bar{x}$ is a local solution of (1.5), (1.1), then

$$
\left\langle f^{\prime}(\bar{x}), h\right\rangle \geq 0 \quad \forall h \in \bar{H}_{2}(\bar{x}) .
$$

If $X$ is finite-dimensional, the right-hand inclusion in (3.2) implies that the following condition is sufficient for $\bar{x}$ to be a strict local solution of our problem:

$$
\left\langle f^{\prime}(\bar{x}), h\right\rangle>0 \quad \forall h \in H_{2}(\bar{x}) \backslash\{0\} .
$$

Dualizing (3.3), we can write that

$$
f^{\prime}(\bar{x}) \in\left(\bar{H}_{2}(\bar{x})\right)^{*},
$$

which is the primal-dual form of necessary optimality conditions. Explicit evaluation of the dual cone in the right-hand side of the above relation in full generality is an extremely difficult problem. However, we are able to give some meaningful results under additional assumptions. Specifically, if cone $K$ is finitely generated and for some $h \in \bar{H}_{2}(\bar{x})$ the inequality in (3.3) holds as an equality, we derive an explicit primal-dual form of necessary optimality conditions. Note that further study of such "critical direction" $h$ is of particular importance in view of the violation of the sufficient optimality condition (3.4). Assumptions of this type are quite common in the literature $[7,8,25]$.

In the proof below, we shall also need the following generalization of the tangent cone description in the regular case. Let, in addition to our standard assumptions, $C$ be a closed finitely generated cone in a normed linear space $Z$, and let $A: X \rightarrow Z$ be a continuous linear operator. Consider the set $\Delta=D \cap E$, where $E=\{x \in X \mid A x \in$ $C\}$, and a point $\bar{x} \in \Delta$. If there exists $\bar{\xi} \in X$ satisfying $A \bar{\xi} \in T_{C}(A \bar{x})$ and Robinson's condition (2.1), then

$$
T_{\Delta}(\bar{x})=\left\{h \in X \mid A h \in T_{C}(A \bar{x}), F^{\prime}(\bar{x}) h \in T_{K}(F(\bar{x}))\right\} .
$$

This generalization is essentially based on the well-known fact that linearity of constraints can be regarded as a special regularity-type assumption.

ThEOREM 3.2. Suppose that the assumptions of Theorem 3.1 are satisfied. Let $K$ be a finitely generated cone, and let the point $\bar{x}$ be a local minimizer for problem (1.5), (1.1). Assume that

$$
\exists h \in \bar{H}_{2}(\bar{x}) \text { such that }\left\langle f^{\prime}(\bar{x}), h\right\rangle=0 .
$$


Then there exist two functionals

$$
y_{1}^{*}=y_{1}^{*}(h) \in K^{*} \cap\{F(\bar{x})\}^{\perp} \cap\left\{F^{\prime}(\bar{x}) h\right\}^{\perp}
$$

and

$$
y_{2}^{*}=y_{2}^{*}(h) \in K^{*} \cap\{F(\bar{x})\}^{\perp} \cap\left(\operatorname{Im} F^{\prime}(\bar{x})\right)^{\perp} \cap\left\{F^{\prime \prime}(\bar{x})[h]^{2}\right\}^{\perp}
$$

such that

$$
f^{\prime}(\bar{x})=\left(F^{\prime}(\bar{x})\right)^{*} y_{1}^{*}+\left(F^{\prime \prime}(\bar{x})[h]\right)^{*} y_{2}^{*} .
$$

Proof. It can be easily seen that there exists a neighborhood $U$ of $h$ in $X$ such that

$$
H_{2}(\bar{x}) \cap U \subset \bar{H}_{2}(\bar{x}) .
$$

(Just recall that since cone $K$ is finitely generated, the second inclusion in (3.1) holds as an equality, and observe that for a neighborhood $U$ small enough, one can choose the same $\bar{h}$ in the definition of $\bar{H}_{2}(\bar{x})$ for all $h \in U$.) Hence, by Theorem 3.1, we have that

$$
\left\langle f^{\prime}(\bar{x}), \xi\right\rangle \geq 0 \quad \forall \xi \in H_{2}(\bar{x}) \cap U .
$$

The latter relation and (3.6) imply that $h$ is a local solution of the optimization problem

$$
\min \left\{\left\langle f^{\prime}(\bar{x}), \xi\right\rangle \mid \xi \in H_{2}(\bar{x})\right\} .
$$

By the classical necessary optimality conditions, it then follows that

$$
\left\langle f^{\prime}(\bar{x}), \xi\right\rangle \geq 0 \quad \forall \xi \in T_{H_{2}(\bar{x})}(h),
$$

or, equivalently,

$$
f^{\prime}(\bar{x}) \in\left(T_{H_{2}(\bar{x})}(h)\right)^{*} .
$$

We now have to evaluate the cone $T_{H_{2}(\bar{x})}(h)$ and its dual. The latter problem is now solvable with the help of Lemma A.4, because our second-order regularity condition with respect to $h$ implies that the cone $T_{H_{2}(\bar{x})}(h)$ is actually given by the linearized model of constraints defining $H_{2}(\bar{x})$. Indeed, using the assumption that cone $K$ is closed and finitely generated, and applying Lemma A.3 and relation (3.5) to appropriate data, we obtain

$$
T_{H_{2}(\bar{x})}(h)=\left\{\begin{array}{l|l}
\xi \in X & \begin{array}{l}
F^{\prime}(\bar{x}) \xi \in K+\operatorname{lin}\{F(\bar{x})\}+\operatorname{lin}\left\{F^{\prime}(\bar{x}) h\right\} \\
F^{\prime \prime}(\bar{x})[h, \xi] \in \operatorname{cl}\left(K+\operatorname{lin}\{F(\bar{x})\}+\operatorname{Im} F^{\prime}(\bar{x})+\operatorname{lin}\left\{F^{\prime \prime}(\bar{x})[h]^{2}\right\}\right)
\end{array}
\end{array} .\right.
$$

Note that cone $K+\operatorname{lin}\{F(\bar{x})\}+\operatorname{lin}\left\{F^{\prime}(\bar{x}) h\right\}$ is closed and finitely generated. Also, $\operatorname{dim} Y<\infty$. (This is implied by our assumption that a finitely generated cone $K$ has nonempty interior.) In particular, it follows that $\operatorname{dim}\left(\operatorname{Im} F^{\prime}(\bar{x})\right)<\infty$. Hence, cone $K+\operatorname{lin}\{F(\bar{x})\}+\operatorname{Im} F^{\prime}(\bar{x})+\operatorname{lin}\left\{F^{\prime \prime}(\bar{x})[h]^{2}\right\}$ is also closed and finitely generated. Now applying Lemma A.4 to (3.11), we obtain the equality

$$
\begin{aligned}
\left(T_{H_{2}(\bar{x})}(h)\right)^{*}= & \left(F^{\prime}(\bar{x})\right)^{*}\left(K^{*} \cap\{F(\bar{x})\}^{\perp} \cap\left\{F^{\prime}(\bar{x}) h\right\}^{\perp}\right) \\
& +\left(F^{\prime \prime}(\bar{x})[h]\right)^{*}\left(K^{*} \cap\{F(\bar{x})\}^{\perp} \cap\left(\operatorname{Im} F^{\prime}(\bar{x})\right)^{\perp} \cap\left\{F^{\prime \prime}(\bar{x})[h]^{2}\right\}^{\perp}\right),
\end{aligned}
$$

from which the conclusion of the theorem follows immediately. 
Theorem 3.2 subsumes classical first-order necessary optimality conditions for the regular case. Indeed, suppose that $h$ in the requirements of Theorem 3.2 satisfies (2.7). Note that this will always be so in the regular case because, by (2.8) and Lemma A.2, the right-hand side of (2.7) coincides with the entire space $Y$. Then, using Lemma A.1, we have that

$$
K^{*} \cap\{F(\bar{x})\}^{\perp} \cap\left(\operatorname{Im} F^{\prime}(\bar{x})\right)^{\perp} \cap\left\{F^{\prime \prime}(\bar{x})[h]^{2}\right\}^{\perp}=\{0\} .
$$

Therefore in that case $y_{2}^{*}=0$, and representation (3.7)-(3.9) reduces to

$$
f^{\prime}(\bar{x})=\left(F^{\prime}(\bar{x})\right)^{*} y_{1}^{*},
$$

with $y_{1}^{*}$ satisfying (3.7). Furthermore, by Remark 2.1, in the regular case Theorem 3.2 can be applied by choosing $h=0$. With this choice, (3.7) takes the form

$$
y_{1}^{*} \in K^{*} \cap\{F(\bar{x})\}^{\perp} .
$$

Combined with feasibility condition $F(\bar{x}) \in K$, relations (3.13), (3.14) coincide with the classical optimality conditions (1.6), where the nonsingular multiplier $y_{0}=1$ is chosen. In terms of the nonlinear programming problem, the inclusion $y_{1}^{*} \in K^{*}$ is the nonnegativity condition for the Lagrange multipliers, and the inclusion $y_{1}^{*} \in\{F(\bar{x})\}^{\perp}$ is the condition of complementary slackness.

As will be shown in section 4, Theorem 3.2 also contains optimality conditions under the second-order regularity of $[7,8]$ but can be applicable when the latter is not.

4. Comparisons and an example. In this section, we provide a comparison of the results obtained above with known approaches to irregular problems, and illustrate our development by an example.

First, we mention Abadie's and Kuhn-Tucker's constraint qualifications (CQs) for nonlinear programming (see [22]; there are also some other CQs of similar type). These are weaker than the Mangasarian-Fromovitz constraint qualification (MFCQ) but still guarantee that the tangent cone is given by the linearized model of the constraints; e.g., see $[23,22]$. From the point of view of the problem data, these CQs are less constructive than MFCQ, which is closer to our development. (MFCQ is subsumed by our framework.) Such CQs of nonalgebraic nature are usually rather difficult to verify directly. Perhaps even more importantly, we deal here with a more general case in which the tangent cone does not necessarily coincide with the linearized cone.

The next issue that deserves to be discussed is reformulating inequality constraints as equalities, with the aim of subsequently using results available for the latter. This technique is known to be useful for regular inequality-constrained problems; e.g., see [9]. Analogously, one might try to apply known optimality conditions for (irregular) equality-constrained problems to reformulations of irregular inequality constraints. For example, the theory of 2-regularity $[29,4,6,5,16,1,13,20,17,15]$ offers optimality conditions for the case in which irregularity of the problem is induced only by equality constraints, with inequality constraints being either absent or regular. We next show that in our context, applicability of this approach is very limited.

For simplicity, let us take $Y=\Re^{m}, K=\Re_{-}^{m}$, and $F(\bar{x})=0$, and reformulate the inequality-constrained set $D$ by introducing slacks:

$$
\Delta=\left\{(x, u) \in X \times \Re^{m} \mid F(x)+u=0, u \geq 0\right\} .
$$


The new set $\Delta$ is given by equality and "simple" inequality constraints. Clearly, the equality constraint in $\Delta$ is regular at every point, but MFCQ is still violated at $(\bar{x}, 0)$. Hence, the classical results for the regular case are not applicable. Results from the theory of 2-regularity are obviously also not useful, as there are simply no irregular equality constraints in $\Delta$.

Another possibility is a purely equality-constrained reformulation:

$$
\Delta=\left\{(x, u) \in X \times \Re^{m} \mid F(x)+u^{2}=0\right\},
$$

where the square is componentwise. Here, the equality constraint is irregular at $(\bar{x}, 0)$, and 2-regularity theory is applicable, at least formally. However, this application leads to something meaningful only when $\operatorname{ker} F^{\prime}(\bar{x}) \neq\{0\}$, which is an unnatural requirement for inequality constraints. Our approach is certainly free of this restriction. Moreover, even if $\operatorname{ker} F^{\prime}(\bar{x}) \neq\{0\}$, for inequality constraints this subspace can have little to do with the tangent cone, as in Example 4.1 below. Without going into detail, we shall mention that there are also some other limitations in the "brute force" approach of applying results known for irregular equality constraints to equation reformulations of irregular inequality constraints. It seems that developing a special approach specifically designed for inequality constraints is really necessary. An initial step in the direction pursued in the present paper was made in [14].

Another known approach to irregular problems consists of second-order necessary and sufficient optimality conditions of Levitin-Milyutin-Osmolovskii type, e.g., $[21,18,7,8,1,2]$ (see also recent work in $[10,25]$ ), which employ F. John first-order necessary conditions (with undefined multiplier corresponding to the objective function). This approach is effective when applied to inequality-constrained problems, but it leads to results of a completely different nature, which makes comparison with the present paper difficult. We note that this approach is not principally associated with precise description of the tangent cone, i.e., it does not deal with sufficient conditions for tangency beyond the regular case.

Next, we discuss the well-known second-order CQ [7,8], which was introduced using second-order parabolic tangent sets, and which is especially relevant for irregular inequality-constrained problems. In our setting, this CQ can be stated as follows:

$$
\begin{aligned}
\exists h \in X \text { such that } & \left\langle f^{\prime}(\bar{x}), h\right\rangle=0, \\
& F^{\prime}(\bar{x}) h \in K+\operatorname{lin}\{F(\bar{x})\}, \\
& F^{\prime \prime}(\bar{x})[h]^{2} \in \operatorname{int} K+\operatorname{lin}\{F(\bar{x})\}+\operatorname{Im} F^{\prime}(\bar{x}) .
\end{aligned}
$$

This condition is also weaker than Robinson's regularity (in the regular case, (4.1)(4.3) hold with $h=0$ ), yet it guarantees that if $\bar{x}$ is a local solution of (1.5), (1.1), then F. John-necessary conditions are satisfied with a nonzero multiplier corresponding to the objective function. Note that relations (4.2) and (4.3) already appear in Theorem 2.2 (see (2.6) and (2.7)), where they are used to explicitly construct a parabolic feasible arc tangent to $h$. But observe that in Theorem 2.2 we consider a larger set of directions. Namely, for an element $h$ satisfying second-order necessary conditions of tangency, this theorem gives constructive sufficient conditions for $h$ to be a limit point of elements satisfying (4.2), (4.3). This is important, because it is certainly possible that (4.1) does not hold for any $h$ satisfying (4.2), (4.3), but that it does hold for some limit point of such elements. Moreover, Example 4.1 below illustrates that this situation (i.e., the second-order CQ (4.1)-(4.3) is violated, but our Theorem 3.2 is applicable) is in fact quite likely to occur. 
Finally, note that if $h$ is an element satisfying (4.1)-(4.3), then (3.6) also holds, and the assumptions of Theorem 3.2 are satisfied. Moreover, in this case, (3.12) holds. Hence, relation (3.8) in Theorem 3.2 implies that $y_{2}^{*}=0$. We conclude that optimality conditions under the second-order CQ (4.1)-(4.3) are a particular case of Theorem 3.2 (under the additional assumption that $K$ is finitely generated).

To complete this section, we present an example illustrating all the results derived above, and showing that they can be applicable when the F. John-optimality conditions and optimality conditions based on classical (first- and second-order) CQs are not useful. Note that our example is not pathological or exotic.

EXAmPle 4.1. Let $X=Y=\Re^{2}, K=\Re_{-}^{2}$, and consider a family of functions

$$
f: \Re^{2} \rightarrow \Re, \quad f(x)=a x_{1}+b x_{2}+\omega_{1}(x)
$$

and the mapping

$$
F: \Re^{2} \rightarrow \Re^{2}, \quad F(x)=\left(-x_{1},-\frac{1}{2}\left(x_{1}^{2}-x_{2}^{2}\right)\right)+\omega_{2}(x),
$$

where $\omega_{1}: \Re^{2} \rightarrow \Re,\left|\omega_{1}(x)\right|=o(\|x\|)$, and $\omega_{2}: \Re^{2} \rightarrow \Re^{2},\left\|\omega_{2}(x)\right\|=o\left(\|x\|^{2}\right)$.

Consider the point $\bar{x}=0$ in $\Re^{2}$. We have that $F(0)=0$, so that $0 \in D$, where $D$ is given by (1.1). It can be easily seen that MFCQ does not hold here, and so classical theory does not apply. By direct computations, we obtain that

$$
\begin{aligned}
& H_{2}(0)=\tilde{H}_{2}(0)=\left\{h \in \mathbf{R}^{2} \mid h_{1} \geq 0, h_{1}^{2}-h_{2}^{2} \geq 0\right\}, \\
\bar{H}_{2}(0)= & \left\{h \in H_{2}(0) \mid \exists \bar{h} \in \mathbf{R}^{2}: \bar{h}_{1} \geq 0, h_{1} \bar{h}_{1}-h_{2} \bar{h}_{2}>0\right\}=H_{2}(0) .
\end{aligned}
$$

Hence, by Theorem 2.2,

$$
T_{D}(0)=H_{2}(0)=\left\{h \in \mathbf{R}^{2}\left|h_{1} \geq\right| h_{2} \mid\right\},
$$

which is actually geometrically obvious. Observe further that the linearized cone is given by

$$
\left\{h \in \mathbf{R}^{2} \mid F^{\prime}(\bar{x}) h \in T_{K}(F(\bar{x}))\right\}=\left\{h \in \mathbf{R}^{2} \mid h_{1} \geq 0\right\},
$$

which is different from $T_{D}(0)$. Hence, the Kuhn-Tucker, Abadie, and any other CQs guaranteeing that the tangent and linearized cones coincide are violated in this example. Note that in this case, the tangent cone is actually polyhedral, just different from the linearized one. This shows that our description can be useful even when the tangent cone is "simple."

It is easy to see that for all values of parameters $a$ and $b$, the F. John conditions (1.6) for problem (1.5), (1.1) hold at 0 with $y_{0}=0$. Furthermore, $y_{0}$ can be nonzero only if $b=0$ and $a \leq 0$. For all other values of the parameters, F. John conditions are not meaningful for describing optimality.

As is easy to see, the set of elements satisfying (4.2), (4.3) is $\left\{h \in \mathbf{R}^{2} \mid h_{1}>\right.$ $\left.\left|h_{2}\right|\right\}$. Clearly, if 0 is a local minimizer, conditions (4.1)-(4.3) can hold for some $h$ simultaneously only if $a=b=0$. Hence, for all other values of the parameters, the classical second-order CQ (4.1)-(4.3) does not hold, and the corresponding results are not applicable.

We next illustrate our approach, considering several characteristic values of the parameters. 
If $a=1, b=-1$, then 0 is a (nonisolated) local minimizer for problem (1.5), (1.1). As is easy to see,

$$
\left\langle f^{\prime}(0), h\right\rangle \geq 0 \quad \forall h \in H_{2}(0),
$$

which illustrates Theorem 3.1. Note that for $h=(1,1) \in H_{2}(0)$, the latter inequality holds as equality, and our primal-dual optimality conditions (3.7)-(3.9) are satisfied with the multipliers

$$
y_{1}^{*}=(0, \alpha) \in \Re^{2}, \quad \alpha \in \Re_{-}, \quad y_{2}^{*}=(0,-1) \in \Re^{2} .
$$

This gives an illustration for Theorem 3.2. Note that for $h \in H_{2}(0) \backslash \operatorname{lin}\{(1,1)\}$, a similar representation does not hold. The reason is that for such $h$, strict inequality holds in (4.4).

If $a=1, b=0$, then (4.4) holds as a strict inequality for every $h \in H_{2}(0) \backslash\{0\}$, and 0 is an isolated local minimizer. This illustrates sufficient optimality condition (3.4).

Finally, if $a=0, b=1$, then it is easy to see that (4.4) does not hold for those elements $h \in H_{2}(0)$ for which $h_{2}<0$. Theorem 3.1 implies that 0 is not a local minimizer in this case. We could similarly use Theorem 3.2 to verify this conclusion. Indeed, for the element $h=(1,0) \in H_{2}(0),(4.4)$ holds as an equality, but there exist no multipliers $y_{1}^{*}, y_{2}^{*} \in \Re^{2}$ for which (3.9) holds.

5. Some further developments. In conclusion, we present some further developments of the optimality conditions obtained above. The first one has to do with a certain form of second-order (in terms of the objective function) necessary optimality conditions, and the second outlines an extension to mixed equality-inequalityconstrained problems.

5.1. Second-order optimality conditions. To derive second-order optimality conditions, we need the following notion. Let $X$ and $\Sigma$ be normed linear spaces, and let a mapping $\Phi: X \rightarrow \Sigma$ be twice Fréchet-differentiable at a point $\bar{x} \in X$. Suppose that $\Sigma_{1}=\operatorname{Im} \Phi^{\prime}(\bar{x})$ is closed and has a closed complementary subspace $\Sigma_{2}$ in $\Sigma$. Let $P$ be a projector onto $\Sigma_{2}$ parallel to $\Sigma_{1}$ in $\Sigma$. (By assumptions above, this projector is continuous.) In this setting, the mapping $\Phi$ is referred to as 2-regular at the point $\bar{x}$ with respect to an element $h \in X$ (see $[29,4,6,5,16,1,13,20,17])$ if

$$
\operatorname{Im}\left(\Phi^{\prime}(\bar{x})+P \Phi^{\prime \prime}(\bar{x})[h]\right)=\Sigma .
$$

We note that the 2-regularity property of $\Phi$ does not depend on a choice of the complementary subspace $\Sigma_{2}$.

The following generalization of the classical Lyusternik's theorem can be found in $[29,5,16,20,17]$.

Proposition 5.1. Let $X$ and $\Sigma$ be Banach spaces. Assume that a mapping $\Phi: X \rightarrow \Sigma$ is three times Fréchet-differentiable at a point $\bar{x} \in X$ such that $\Phi(\bar{x})=0$. Assume further that $\Phi$ is 2-regular at $\bar{x}$ with respect to an element $h \in X$ such that

$$
h \in \operatorname{Ker} \Phi^{\prime}(\bar{x}), \quad \Phi^{\prime \prime}(\bar{x})[h]^{2} \in \operatorname{Im} \Phi^{\prime}(\bar{x}) .
$$

Then there exist a number $\delta>0$ and a mapping $r:(-\delta, \delta) \rightarrow X$ such that

$$
\Phi(\bar{x}+t h+r(t))=0 \quad \forall t \in(-\delta, \delta), \quad\|r(t)\|=O\left(t^{2}\right) .
$$


We next derive a special form of higher-order necessary optimality conditions using the results obtained in section 3 .

THEOREM 5.2. Let $X$ and $Y$ be Banach spaces, let $K$ be a closed finitely generated cone in $Y$ with a nonempty interior, and let $f: X \rightarrow \Re$ be twice and $F: X \rightarrow Y$ be three times Fréchet-differentiable at the point $\bar{x}$, which is a local minimizer for problem (1.5), (1.1). Assume that (3.6) holds, and let $\tilde{\Pi}$ be a (continuous) projector onto some closed complementary subspace $\tilde{Y}$ of $\operatorname{lin}\left\{F(\bar{x}), F^{\prime}(\bar{x}) h\right\}$ in $Y$. Assume finally that

$$
\tilde{\Pi} F^{\prime \prime}(\bar{x})[h]^{2} \in \tilde{\Pi} \operatorname{Im} F^{\prime}(\bar{x})
$$

and that the mapping $\Phi: X \rightarrow \tilde{Y}, \Phi(x)=\tilde{\Pi} F(x)$, is 2-regular at the point $\bar{x}$ with respect to $h$. Then for every $y_{1}^{*}, y_{2}^{*} \in Y^{*}$ satisfying (3.7)-(3.9), it holds that

$$
f^{\prime \prime}(\bar{x})[h]^{2}-\left\langle y_{1}^{*}, F^{\prime \prime}(\bar{x})[h]^{2}\right\rangle-\frac{1}{3}\left\langle y_{2}^{*}, F^{\prime \prime \prime}(\bar{x})[h]^{3}\right\rangle \geq 0 .
$$

Proof. By the definition of $\tilde{\Pi}$, we have

$$
\Phi^{\prime}(\bar{x}) h=\tilde{\Pi} F^{\prime}(\bar{x}) h=0 .
$$

Hence, taking into account (5.1), Proposition 5.1 is applicable (with $\Sigma=\tilde{Y}$ ). So for some number $\delta>0$ and some mapping $r:(-\delta, \delta) \rightarrow X$, we have that $\forall t \in(-\delta, \delta)$

$$
\tilde{\Pi} F(\bar{x}+t h+r(t))=0, \quad\|r(t)\|=O\left(t^{2}\right),
$$

where the first equality means that

$$
F(\bar{x}+t h+r(t)) \in \operatorname{lin}\left\{F(\bar{x}), F^{\prime}(\bar{x}) h\right\} .
$$

By (3.7), $y_{1}^{*} \in\left(\operatorname{lin}\left\{F(\bar{x}), F^{\prime}(\bar{x}) h\right\}\right)^{\perp}$. Hence, $\forall t \in(-\delta, \delta)$ we have

$$
\begin{aligned}
0 & =\left\langle y_{1}^{*}, F(\bar{x}+t h+r(t))\right\rangle \\
& =\left\langle y_{1}^{*}, F^{\prime}(\bar{x}) r(t)\right\rangle+\frac{1}{2}\left\langle y_{1}^{*}, F^{\prime \prime}(\bar{x})[t h]^{2}\right\rangle+o\left(t^{2}\right) .
\end{aligned}
$$

Similarly, by (3.8), $y_{2}^{*} \in\left(\operatorname{lin}\left\{F(\bar{x}), F^{\prime}(\bar{x}) h\right\}\right)^{\perp}$ and also $y_{2}^{*} \in\left(\operatorname{Im} F^{\prime}(\bar{x})\right)^{\perp}$, which implies that

$$
\begin{aligned}
0 & =\left\langle y_{2}^{*}, F(\bar{x}+t h+r(t))\right\rangle \\
& =\left\langle y_{2}^{*}, F^{\prime \prime}(\bar{x})[t h, r(t)]\right\rangle+\frac{1}{6}\left\langle y_{2}^{*}, F^{\prime \prime \prime}(\bar{x})[t h]^{3}\right\rangle+o\left(t^{3}\right) .
\end{aligned}
$$

By (5.3), there exist $\lambda_{1}, \lambda_{2}:(-\delta, \delta) \rightarrow \Re$ such that

$$
F(\bar{x}+t h+r(t))=\lambda_{1}(t) F(\bar{x})+\lambda_{2}(t) F^{\prime}(\bar{x}) h .
$$

On the other hand, by differentiability of $F$,

$$
F(\bar{x}+t h+r(t))=F(\bar{x})+t F^{\prime}(\bar{x}) h+o(t) .
$$

Therefore, we can take $\lambda_{1}(t)=1+o(t), \lambda_{2}(t)=t+o(t)$. Since $h \in \tilde{H}_{2}(\bar{x})$, we have that $F^{\prime}(\bar{x}) h=y+\lambda F(\bar{x})$ for some $y \in K, \lambda \in \Re$. We further obtain

$$
\begin{aligned}
F(\bar{x}+t h+r(t)) & =(1+o(t)) F(\bar{x})+(t+o(t))(y+\lambda F(\bar{x})) \\
& =(1+\lambda t+o(t)) F(\bar{x})+(t+o(t)) y .
\end{aligned}
$$


Taking into account that $F(\bar{x}) \in K$ and $y \in K$, it is clear now that if $\delta>0$ is small enough, then $\bar{x}+t h+r(t) \in D \forall t \in(0, \delta)$, and since $\bar{x}$ is a local minimizer, by differentiability of $f$ it follows that $\forall t \in(0, \delta)$

$$
0 \leq f(\bar{x}+t h+r(t))-f(\bar{x})=\left\langle f^{\prime}(\bar{x}), r(t)\right\rangle+\frac{1}{2} f^{\prime \prime}(\bar{x})[t h]^{2}+o\left(t^{2}\right),
$$

where we have also used (3.6). Combining the latter relation with (5.4) and (5.5) (divided by -1 and $-t$, respectively), we obtain

$$
\begin{aligned}
0 \leq & \left\langle f^{\prime}(\bar{x}), r(t)\right\rangle-\left\langle y_{1}^{*}, F^{\prime}(\bar{x}) r(t)\right\rangle-\left\langle y_{2}^{*}, F^{\prime \prime}(\bar{x})[h, r(t)]\right\rangle \\
& +\frac{1}{2} f^{\prime \prime}(\bar{x})[t h]^{2}-\frac{1}{2}\left\langle y_{1}^{*}, F^{\prime \prime}(\bar{x})[t h]^{2}\right\rangle-\frac{1}{6 t}\left\langle y_{2}^{*}, F^{\prime \prime \prime}(\bar{x})[t h]^{3}\right\rangle+o\left(t^{2}\right) \\
= & \left\langle f^{\prime}(\bar{x})-\left(F^{\prime}(\bar{x})\right)^{*} y_{1}^{*}-\left(F^{\prime \prime}(\bar{x})[h]\right)^{*} y_{2}^{*}, r(t)\right\rangle \\
& +\frac{t^{2}}{2}\left(f^{\prime \prime}(\bar{x})[h]^{2}-\left\langle y_{1}^{*}, F^{\prime \prime}(\bar{x})[h]^{2}\right\rangle-\frac{1}{3}\left\langle y_{2}^{*}, F^{\prime \prime \prime}(\bar{x})[h]^{3}\right\rangle\right)+o\left(t^{2}\right) \\
= & \frac{t^{2}}{2}\left(f^{\prime \prime}(\bar{x})[h]^{2}-\left\langle y_{1}^{*}, F^{\prime \prime}(\bar{x})[h]^{2}\right\rangle-\frac{1}{3}\left\langle y_{2}^{*}, F^{\prime \prime \prime}(\bar{x})[h]^{3}\right\rangle\right)+o\left(t^{2}\right),
\end{aligned}
$$

where the last equality follows from (3.9). Dividing by $t^{2} / 2$ and passing onto the limit as $t \rightarrow 0$, we obtain (5.2).

Note that the mapping $\Phi$ defined in Theorem 5.2 could be regular (rather than 2-regular) only if the Robinson's regularity condition were to be satisfied at $\bar{x}$.

The next example illustrates that Theorem 5.2 provides additional information that can be used to eliminate candidates for optimality.

EXAmple 5.1. Consider the setting of Example 4.1, where $a=1, b=-1$, $\omega_{2}(\cdot) \equiv 0$ on $\Re^{2}$, and $\omega_{1}: \Re^{2} \rightarrow \Re$ is a quadratic form negative on $h=(1,1)$. Then the first-order necessary conditions given by Theorems 3.1 and 3.2 are satisfied at 0 (see Example 4.1), but by direct inspection it can be seen that the second-order necessary optimality conditions given by Theorem 5.2 are violated. Indeed, $F^{\prime}(\bar{x}) h=(-1,0)$, and so one can take $\tilde{Y}=\operatorname{lin}\{(0,1)\}$. Then $\Phi$ can be considered as a scalar-valued function

$$
\Phi: \Re^{2} \rightarrow \Re, \quad \Phi(x)=-\frac{1}{2}\left(x_{1}^{2}-x_{2}^{2}\right) .
$$

This function is certainly 2-regular at 0 with respect to every nonzero element. (For scalar-valued functions, the latter property is equivalent to saying that 0 is a nondegenerate critical point [3].) In particular, $\Phi$ is 2 -regular at 0 with respect to $h$, which obviously satisfies (5.1.) We further have that

$$
f^{\prime \prime}(\bar{x})[h]^{2}-\left\langle y_{1}^{*}, F^{\prime \prime}(\bar{x})[h]^{2}\right\rangle-\frac{1}{3}\left\langle y_{2}^{*}, F^{\prime \prime \prime}(\bar{x})[h]^{3}\right\rangle=2 \omega_{1}(h)<0,
$$

which is in contradiction with (5.2). We conclude that 0 is not a local minimizer for problem (1.5), (1.1).

5.2. Mixed equality and inequality constraints. In contrast to the regular case, it appears very difficult (if not impossible) to extend the results for irregular equality- or inequality-constrained problems to the case with mixed inequality and equality constraints, except for some special cases. (For a complete modification of this kind, one would have to avoid the condition that cone $K$ has a nonempty 
interior.) One special case, specifically where the singularity/irregularity is due to equality-type constraints only, is studied thoroughly in $[5,20]$ (those results were already mentioned in section 4). Let us consider briefly the opposite case, i.e., where irregularity is induced by inequality constraints, while equality constraints are regular. Let set $D$ now be given by

$$
D=\{x \in X \mid F(x) \in K, G(x)=0\} .
$$

Assume $G: X \rightarrow Z$ is three times continuously differentiable, where $X$ and $Z$ are Banach spaces. Suppose $G$ is regular at a point $\bar{x} \in D$, i.e.,

$$
\operatorname{Im} G^{\prime}(\bar{x})=Z,
$$

and there exists a continuous projector $\Pi$ on $\operatorname{Ker} G^{\prime}(\bar{x})$ in $Z$. According to the classical facts of nonlinear analysis (see, e.g., $[3,13]$ ), under those assumptions there exist a neighborhood $U$ of 0 in $X$ and a mapping $\rho: U \rightarrow X$ such that $\rho(0)=\bar{x}, \rho(U)$ is a neighborhood of $\bar{x}$ in $X, \rho$ is a $C^{3}$-diffeomorphism from $U$ onto $\rho(U)$, and

$$
\begin{gathered}
G(\rho(x))=G^{\prime}(\bar{x}) x \quad \forall x \in U, \\
\rho^{\prime}(x)=(R(x))^{-1} R(0) \quad \forall x \in U,
\end{gathered}
$$

where

$$
R(x): X \rightarrow Y \times \operatorname{Ker} G^{\prime}(\bar{x}), \quad R(x) \xi=\left(G^{\prime}(\rho(x)) \xi, \Pi \xi\right), \quad x \in U .
$$

Now instead of a feasible point $\bar{x}$ of problem (1.5), (5.6), we can consider for local analysis the feasible point 0 of the inequality-constrained problem

$$
\min \{\varphi(x) \mid x \in \Delta\}, \quad \Delta=\{x \in \tilde{X} \cap U \mid \Phi(x) \in K\},
$$

where $\tilde{X}=\operatorname{Ker} G^{\prime}(\bar{x})$,

$$
\varphi(x)=f(\rho(x)), \quad \Phi(x)=F(\rho(x)), \quad x \in U .
$$

Note that taking advantage of (5.7), it is easy to obtain explicit formulas for the first three derivatives of $\varphi$ and $\Phi$, and so the analysis developed in this paper is applicable to the derivation of optimality conditions for problem (1.5), (5.6).

Appendix. Auxiliary results. All results in this section can be found in standard books on convex analysis $[28,3,19,26]$ or follow from results contained therein.

Lemma A.1. Let $\Sigma$ be a topological linear space, $L$ be a linear subspace in $\Sigma$, and $C$ be a convex cone in $\Sigma$ such that $\operatorname{int} C \neq \emptyset$. Then

$$
\operatorname{int} C \cap L=\emptyset \quad \Leftrightarrow \quad C^{*} \cap L^{\perp} \neq\{0\} .
$$

Lemma A.2. Let $\Sigma$ be a topological linear space and $\Omega_{1}, \Omega_{2}$ be convex sets in $\Sigma$, with int $\Omega_{1} \neq \emptyset$. Then

$$
\operatorname{int}\left(\Omega_{1}+\Omega_{2}\right)=\operatorname{int} \Omega_{1}+\Omega_{2} .
$$

Lemma A.3. Let $\Sigma$ be a normed linear space and $C_{1}, C_{2}$ be finitely generated cones in $\Sigma$. Then

$$
\operatorname{cl}\left(C_{1}+C_{2}\right)=\operatorname{cl} C_{1}+\operatorname{cl} C_{2} .
$$


Lemma A.4. Let $\Upsilon$ and $\Sigma$ be normed linear spaces, $\operatorname{dim} \Sigma<\infty, \Lambda: \Upsilon \rightarrow \Sigma$ be a continuous linear operator, and $C$ be a nonempty closed finitely generated cone in $\Sigma$. Then for a cone $\Gamma=\{\xi \in \Upsilon \mid \Lambda \xi \in C\}$ it holds that

$$
\Gamma^{*}=\Lambda^{*} C^{*} .
$$

Lemma A.5. Let $\Sigma$ be a locally convex topological linear space, and $C$ be a convex cone in $\Sigma$. Then

$$
\sigma^{1} \in \operatorname{cl} C, \sigma^{2} \in \operatorname{int} C \quad \Rightarrow \quad \sigma^{1}+\sigma^{2} \in \operatorname{int} C .
$$

Acknowledgments. We are grateful to the two anonymous referees and the editor for constructive suggestions which led to considerable improvement of the paper.

\section{REFERENCES}

[1] A. V. Arutyunov, Extremum Conditions. Abnormal and Degenerate cases (in Russian), Factorial, Moscow, Russia, 1997.

[2] A. V. Arutyunov, Second-order conditions in extremal problems: The abnormal points, Trans. Amer. Math. Soc., 350 (1998), pp. 4341-4365.

[3] J. P. Aubin and I. Ekeland, Applied Nonlinear Analysis, Wiley, New York, 1984.

[4] Y. R. Avakov, Extremum conditions for smooth problems with equality-type constraints, USSR Comput. Math. and Math. Phys., 25 (1995), pp. 24-32.

[5] Y. R. Avakov, Necessary extremum conditions for smooth abnormal problems with equalityand inequality constraints, J. Math. Notes, 45 (1989), pp. 431-437.

[6] K. N. Belash and A. A. Tretyakov, Methods for solving singular problems, Comput. Math. Math. Phys., 28 (1988), pp. 1097-1102.

[7] J. F. BEN-TAL, Second-order and related extremality conditions in nonlinear programming, J. Optim. Theory Appl., 31 (1980), pp. 143-165.

[8] J. F. BEN-TAL AND J. Zowe, A unified theory of first and second order optimality conditions for extremum problems in topological vector spaces, Math. Programming Study, 19 (1982), pp. 39-76.

[9] D. P. Bertsekas, Constrained Optimization and Lagrange Multiplier Methods, Academic Press, New York, 1982.

[10] J. F. Bonnans, R. Cominetti, And A. Shapiro, Second order optimality conditions based on parabolic second order tangent sets, SIAM J. Optim., 9 (1999), pp. 466-492.

[11] J. F. Bonnans And A. Shapiro, Optimization problems with perturbations: A guided tour, SIAM Rev., 40 (1998), pp. 228-264.

[12] J. F. Bonnans And A. Shapiro, Perturbation Analysis of Optimization Problems, SpringerVerlag, New York, 2000.

[13] A. F. Izmailov, On certain generalizations of Morse's lemma, Proc. Steklov Inst. Math., 220 (1998), pp. 138-153.

[14] A. F. Izmailov, Optimality conditions for degenerate extremum problems with inequality-type constraints, Comput. Math. Math. Phys., 34 (1994), pp. 723-736.

[15] A. F. Izmailov And M. V. Solodov, The theory of 2-regularity for mappings with Lipschitzian derivatives and its applications to optimality conditions, Math. Oper. Res., to appear.

[16] A. F. Izmailov and A. A. Tretyakov, Factor analysis of Nonlinear Mappings (in Russian), Nauka, Moscow, Russia, 1994.

[17] A. F. Izmailov and A. A. Tretyakov, 2-Regular Solutions of Nonlinear Problems. Theory and Numerical Methods (in Russian), Fizmatlit, Moscow, Russia, 1999.

[18] A. D. Ioffe, Necessary and sufficient conditions for a local minimum, 3: Second order conditions and augmented duality, SIAM J. Control Optim., 17 (1979), pp. 266-288.

[19] A. D. Ioffe And V. M. Tikhomirov, Theory of Extremal Problems, North-Holland, Amsterdam, The Netherlands, 1974.

[20] U. LedZewicz AND H. SchätTLER, High-order approximations and generalized necessary conditions for optimality, SIAM J. Control Optim., 37 (1998), pp. 33-53.

[21] E. S. Levitin, A. A. Milyutin, And N. P. Osmolovskil, Conditions of higher order for a local minimum in problems with constraints, Russian Math. Surveys, 33 (1978), pp. 97-168.

[22] O. L. Mangasarian, Nonlinear Programming, McGraw-Hill, New York, 1969. 
[23] O. L. Mangasarian and S. Fromovitz, The Fritz John necessary optimality conditions in the presence of equality and inequality constraints, J. Math. Anal. Appl., 7 (1967), pp. 37-47.

[24] J.-P. Penot, On regularity conditions in mathematical programming, Math. Programming Study, 19 (1982), pp. 167-199.

[25] J.-P. Penot, Second-order conditions for optimization problems with constraints, SIAM J. Control Optim., 37 (1999), pp. 303-318.

[26] B. N. Pshenichniy, Necessary Conditions of an Extremum (in Russian), Nauka, Moscow, Russia, 1982.

[27] S. M. RobInson, Stability theorems for systems of inequalities, Part II: Differentiable nonlinear systems, SIAM J. Numer. Anal., 13 (1976), pp. 497-513.

[28] R. T. Rockafellar, Convex Analysis, Princeton University Press, Princeton, NJ, 1970.

[29] A. A. Tretyakov, Necessary and sufficient conditions for optimality of p-th order, Comput. Math. Math. Phys., 24 (1984), pp. 123-127. 